\title{
Wireless Sensor Networks Projects in a Computer Engineering Program
}

\section{Dr. Afsaneh Minaie, Utah Valley University}

Afsaneh Minaie is a professor of Computer Engineering at Utah Valley University. She received her B.S., M.S., and Ph.D. all in Electrical Engineering from University of Oklahoma. Her research interests include gender issues in the academic sciences and engineering fields, Embedded Systems Design, Mobile Computing, Wireless Sensor Networks, and Databases.

\section{Dr. Reza Sanati-Mehrizy, Utah Valley University}

Reza Sanati-Mehrizy is a professor of Computer Science Department at Utah Valley University, Orem, Utah. He received his M.S. and Ph.D. in Computer Science from the University of Oklahoma, Norman, Oklahoma. His research focuses on diverse areas such as: Database Design, Data Structures, Artificial Intelligence, Robotics, Computer Aided Manufacturing, Data Mining, Data Warehousing, and Machine Learning.

\section{Mr. Paymon Sanati-Mehrizy, Icahn School of Medicine at Mount Sinai}

Paymon is currently a medical student at the Icahn School of Medicine at Mount Sinai. He completed his undergraduate studies in Biology from the University of Pennsylvania in May 2012. Currently, his research interests consist of higher education curricula, both with universities and medical schools.

\section{Dr. Ali Sanati-Mehrizy}

Dr. Ali Sanati-Mehrizy is a Pediatric resident physician at Rutgers University - New Jersey Medical School in Newark, NJ. He is a graduate of the Milton S. Hershey Pennsylvania State University College of Medicine. He completed his undergraduate studies in Biology from the University of Utah. His research interests are varied and involve pediatric hematology and oncology as well as higher education curricula, both with universities and medical schools. 


\title{
Wireless Sensor Networks Projects in a Computer Engineering Program
}

\begin{abstract}
Wireless Sensor Networks (WSNs) are possibly one of the most important technologies of this century and have the potential to make human life more comfortable. WSNs have emerged as an effective solution applied to a wide array of problems. The increasing popularity of WSN has motivated computer engineering programs to provide students with a foundation in the area. In order to integrate wireless sensor networks concepts into our computer engineering curriculum, we have decided to integrate wireless sensor concepts in our Embedded Systems Design II course. A requirement for this course is to write a research paper on the topic of Wireless Sensor Networks. As a result of this assignment, many students have started to do their senior design project on this subject. This paper presents the details of two wireless sensor networks projects that our computer engineering students have done in their senior capstone course.
\end{abstract}

\section{Introduction}

Rapid advances in digital electronics, wireless communications, and Micro Electro-Mechanical Systems (MEMS) technology have led to the emergence of Wireless Sensor networks (WSNs). A WSN consists of spatially distributed autonomous sensors that cooperate by monitoring physical or environmental conditions, such as temperature, sound, light, vibration, motion, pressure, or pollutants ${ }^{1}$. These networks consist of small battery-operated sensor nodes that feature limited computational and radio communication capabilities. Each sensor node in a sensor network consists of three subsystems: the sensor which senses the environment, the processing subsystem which performs local computations on the sensory data collected, and the communication subsystem which is responsible for communication with neighboring sensor nodes ${ }^{8}$.

A wireless sensor network consists of a large number of wireless-capable sensor devices working collaboratively to achieve a shared goal ${ }^{8}$. A WSN may have one or multiple base-stations which collect data from all sensory devices. These base-stations serve as the interface through which the WSN interacts with the outside world ${ }^{3}$. The basic premise of a WSN is to perform networked sensing using a large number of relatively rudimentary sensors instead of utilizing the more conventional approach of developing a few expensive and sophisticated sensing modules. ${ }^{3}$ The potential advantage of networked sensing over the conventional approach, can be summarized as greater coverage, accuracy and reliability at a possibly lower $\operatorname{cost}^{3,8}$.

“The concept of WSN was proposed by the U.S. military as early as the 1970's". 6 In 2003, MIT's Technology Review stated that the WSN is one of the top ten emerging technologies for the $21^{\text {st }}$ century and has been developing at an accelerated pace in the past ten years ${ }^{6}$. Since wireless sensor networks are becoming increasingly popular, much research has been performed in order to advance the technology. However, many challenges still remain for wireless sensor networks. These challenges include possibly unreliable wireless communication systems, limited power availability, failure of nodes, and large- scale deployment. ${ }^{8}$ 
Wireless Sensor Network is an active area of research with various applications. Some of the applications of WSNs includes homeland security, environmental monitoring, safety, health care system, monitoring of space assets for potential and human-made threats in space, ground-based monitoring of both land and water, intelligence gathering for defense, precision agriculture, , civil structure monitoring, urban warfare, weather and climate analysis and prediction, battlefield monitoring and surveillance, exploration of the Solar System and beyond, monitoring of seismic acceleration, temperature, wind speed and GPS data ${ }^{2,8}$. For each application area, there are different technical issues that researchers are currently resolving. Open research issues and challenges are identified to spark new interests and developments in this field ${ }^{8}$. However, the design of wireless sensor networks introduces formidable challenges, since the required body of knowledge encompasses a wide range of topics in the field of electrical and computer engineering, as well as computer science ${ }^{4,8}$.

Educational excellence requires exposing students to the current edge of research. In order to ensure student projects remain along the same trajectory as their respective industries, educators must continually introduce emerging techniques, practices, and applications into the curriculum. The field of wireless sensor networks is growing quickly, and there is an increasing interest in providing students with a foundation in the area. It is crucial that the emerging field of wireless sensor networks be integrated into the computer engineering curriculum ${ }^{8}$.

\section{Utah Valley University}

Utah Valley University is a regional teaching university in Utah with an enrollment of over 31,500 (Fall 2012). Established in 1941, first as a technical college, then a community college, the institution became a state college in 1993 and a regional university in 2008. UVU retains much of the mission and philosophy of a community college in its lower division courses, offering 63 associate degree programs in addition to 67 bachelors and 3 masters programs. The Computer Engineering program at UVU is the first and only engineering program at this institution. It is housed in the Computer Science Department. In our Computer Science Department at UVU, there are three programs, which include Computer Engineering, Software Engineering, and Computer Science. Our Computer Science Program has two areas of specialization, including the traditional computer science area in addition to computer networking. Our Computer Engineering program was approved by the institutional Board of Trustees on April 11, 2002 and was approved by Board of Regents on July 2012. It took us ten years to get approval for this program. Before receiving this approval, Computer Engineering was an area of specialization in the computer science program.

\section{Computer Engineering Senior Design Project Course at UVU}

The purpose of a capstone design project course is to provide graduating senior students the opportunity to demonstrate understanding of the concepts they have learned during the course of their studies. As with many computer engineering programs, students of the computer engineering program at Utah Valley University conclude their degree programs with a semester capstone design experience. The intent is for students to utilize competencies developed in the first three years of the curriculum in the solution of an embedded design problem. Our senior design course is structured as a collection of independent student projects. 
This course is offered every semester. The students in the Computer Engineering program take this course during their last semester. Students either can come up with an embedded project independently or work on a project that is given to them by their advisors. Students write a proposal to define problems and identify solution approaches for their project and the hardware and software that is needed for their project. After several iterations, the advisor approves their project. The faculty adviser will meet with each student individually on a weekly basis at a regularly scheduled, mutually agreeable time. At each meeting, issues associated with the project will be discussed and a status report will be provided to the advisor. Students will keep a daily journal/work log detailing the work that was done, how much time was spent that day, and any technical details that might be needed for later reference. Faculty advisor keeps notes of each meeting as well as action items to be accomplished for the next meeting. Reviewing the log sheet from the previous meeting is a great way for the faculty to prepare for the upcoming one, and provides further evidence to the student of the meeting's importance. At the end of the semester, they turn in a final written report and a final presentation which is evaluated by several faculty members from the department.

\section{Integration of Wireless Sensor Networks in Computer Engineering Program at UVU}

In order to prepare our computer engineering students for the embedded systems design experience, which serves a critical element of their education; we offer two courses on embedded system design. Embedded Systems I is a junior level course and Embedded Systems II is a senior level course. However, these two courses on embedded systems design are not sufficient to teach the students the skills that they need. To satisfy the ABET requirements, our students are required to take a capstone design course. The focus of our computer engineering capstone design class has been the design of embedded systems. By requiring an embedded design project in our capstone course, our students receive hands-on training in embedded systems that will enable them after graduation ${ }^{12}$.

To integrate wireless sensor networks concepts into our curriculum, we decided to use the third $\operatorname{model}^{8}$ and integrated wireless sensor concepts in our Embedded Systems Design II course. In order to interest students in the subject of Wireless Sensor networks, they are required to write a research paper on the topic of Wireless Sensor Networks in the Embedded Systems Design II course. As a result of this assignment, many students have started to do their senior design project on this subject. This paper presents the details of two wireless sensor networks projects that our computer engineering students have done in their capstone course.

\section{First Project: Simulation of Wireless Sensor Networks Utilizing Sun SPOTs Project}

The objective of this project was to simulate a wireless sensor network using the Sun SPOT (Small Programmable Object Technology) as motes.

Sun SPOTs are small, Java-based, wireless devices developed by Sun Microsystems in $2007^{9}$. The device uses the IEEE 802.15.4 standards for its networking. Sun SPOTs are powered by a specially designed small-footprint java virtual machine, called Squawk that can host multiple applications concurrently. Squawk runs directly on the processor without an operating system. 
Because of its Java implementation, programming the Sun SPOT is easy. All of the Sun SPOT SDK and the hardware are available as open source from java.net. A completely assembled Sun SOPT is very small and fits in the palm of a hand.

The Sun SPOT Java Development Kit includes a basestation and two Sun SPOT devices (free range Sun SPOTs) equipped with sensor boards and rechargeable batteries. The Sun SPOT devices units includes an accelerometer, temperature and light sensors, radio transmitter, eight multicolored LEDs, 2 push-button control switches, 5 digital I/O pins, 6 analog inputs, 4 digital outputs, and a rechargeable battery. The Sun SPOT devices dimensions in $\mathrm{mm}$ are roughly $71 \times 42 \times 23$ and each weighs approximately 54 grams. The base station contains a main board and is powered through a USB cable connected to a computer. Its main purpose is to act as a radio gateway between the other two Sun SPOT devices and the host workstation. The two free range Sun SPOTs consist of a battery pack, a processor board (main board), and a sensor board (eDemo Board) as shown in Figure 1.

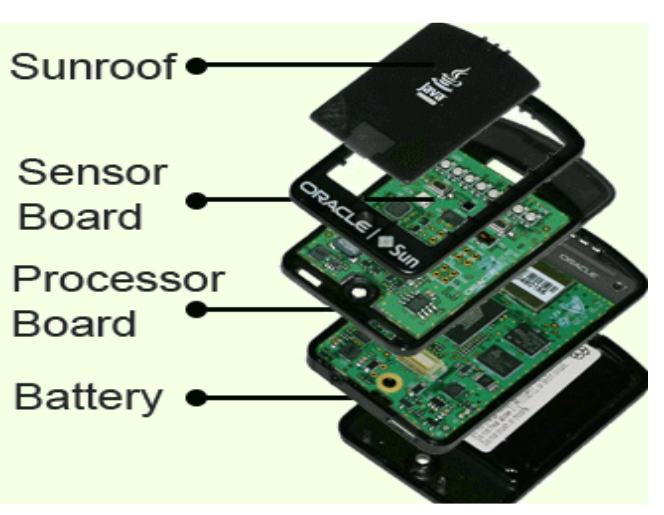

Figure 1: Free Range Sun SPOT ${ }^{9}$

The two free range Sun SPOTs come with a rechargeable battery for use in the field as well as connections to be powered by an external source. Figure 2, illustrates the schematic of connections between boards and battery for a Sun SPOT device. The eSPOT Main Board is the brain of the Sun SPOT system. The eSPOT Main Board contains the main processor, the main board communication, memory, wireless radio, and power controller. The eDEMO board contains the sensors. Figure 3 illustrates the block diagram of the eSPOT Main Board. 


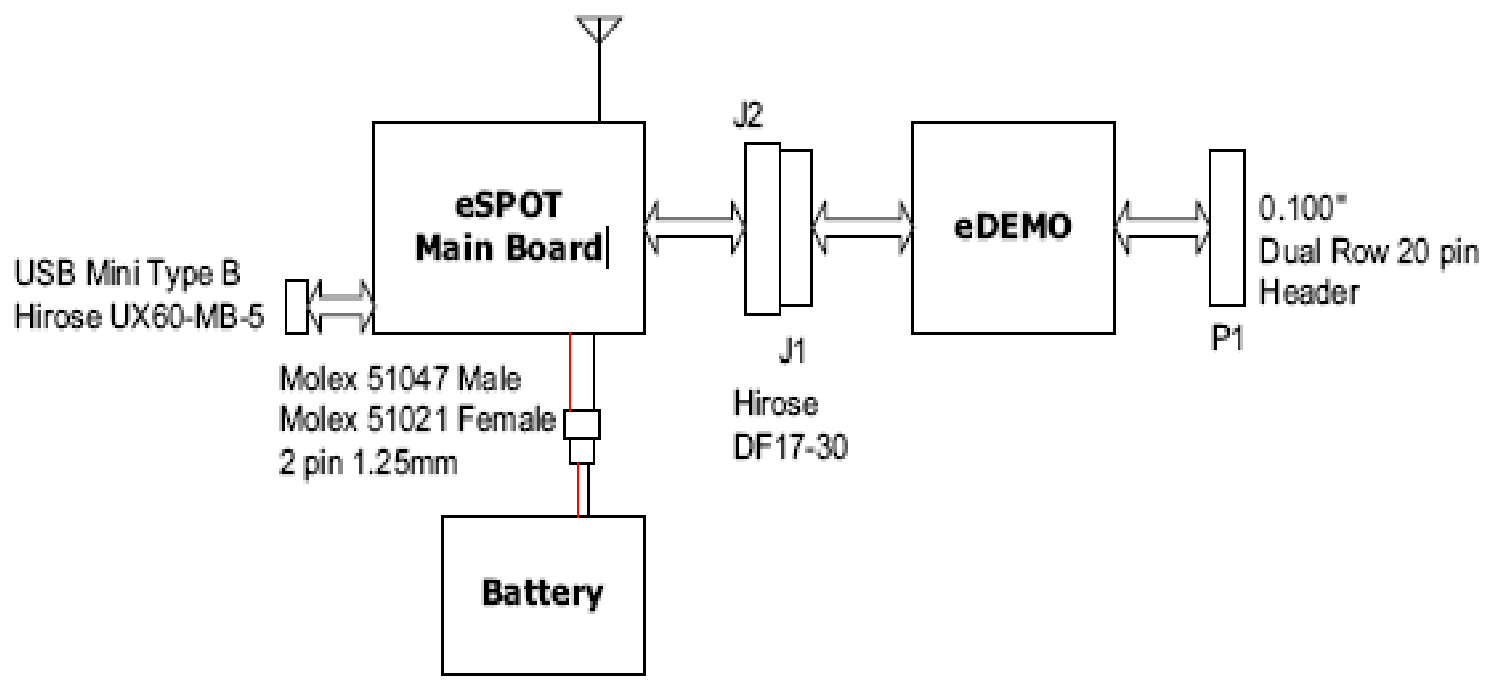

Figure 2: Block Diagram of a Free Sun SPOT ${ }^{9}$

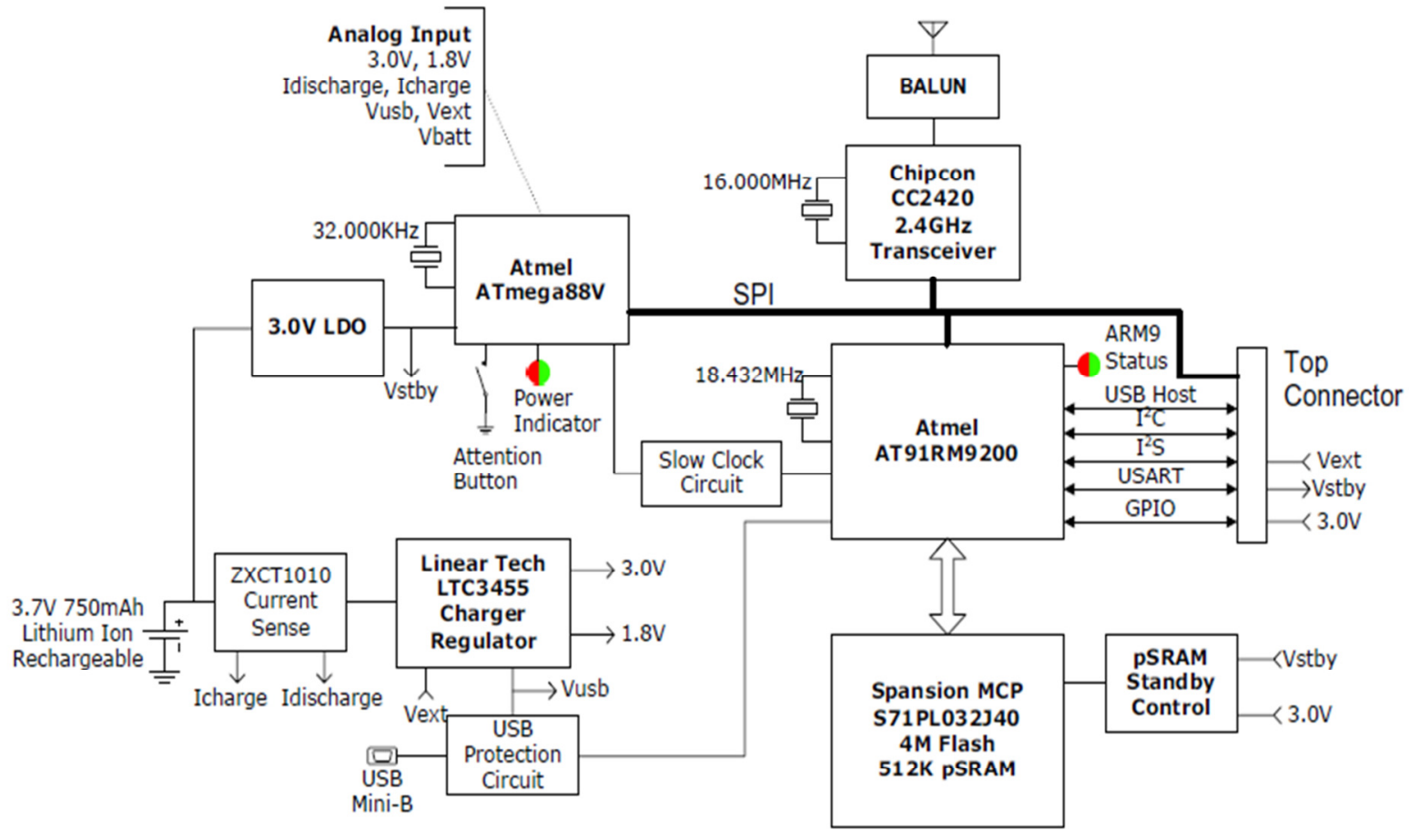

Figure 3: eSPOT Main Board ${ }^{9}$

To simulate a WSN, a simple car was designed by the student to carry one of the free range Sun SPOTs in the search of the second free range Sun SPOT. The free range Sun SPOT which was on the car also communicated to the basestation the room temperature and the light intensity. Figure 4 illustrates a block diagram of the design. 


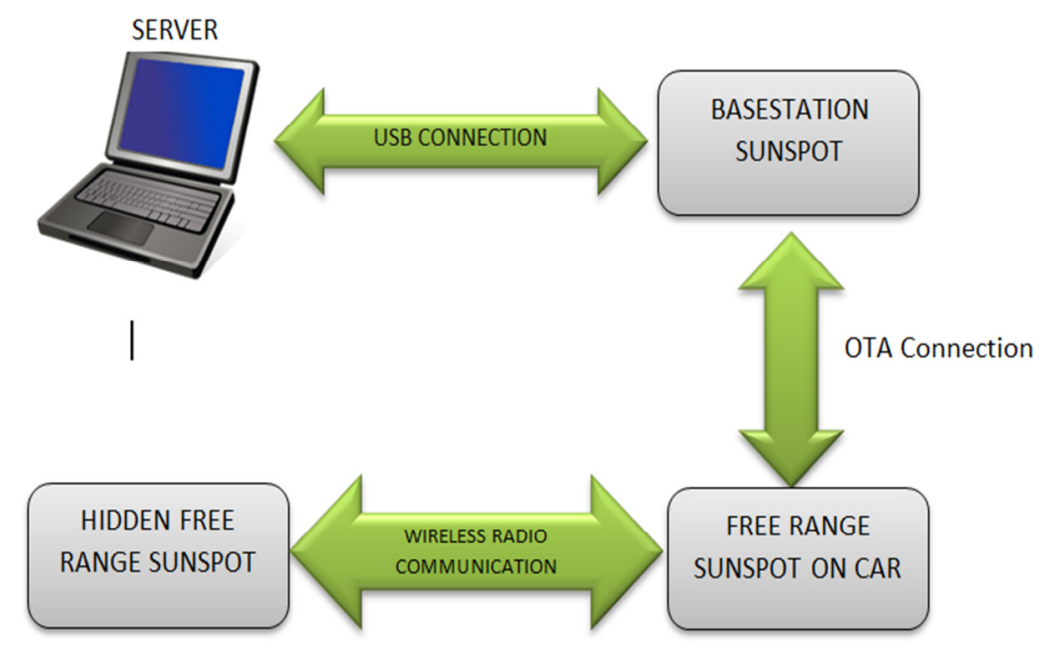

Figure 4: Block Diagram of the $\mathrm{WSN}^{10}$

Figure 5 illustrates the small car which is controlled by one of the free range Sun SPOTs. The Sun SPOTs are capable of generating PWM signals. This feature is used to control the servos of the car. The following code fragment is used to setup the servo motors with $\mathrm{H} 0$ and $\mathrm{H} 1$ pins:

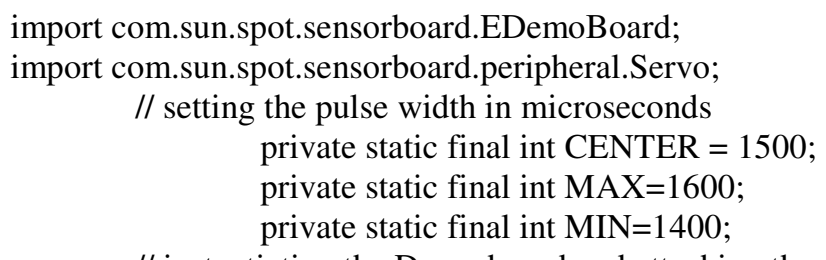

// instantiating the Demo board and attaching the output pins EDemoBoard demo $=$ EDemoBoard.getInstance();

Servo leftservo $=$ new Servo(demo.getOutputPins() $[$ EDemoBoard.H0]);

Servo rightservo $=$ new Servo(demo.getOutputPins()[EDemoBoard.H1] $){ }^{10}$

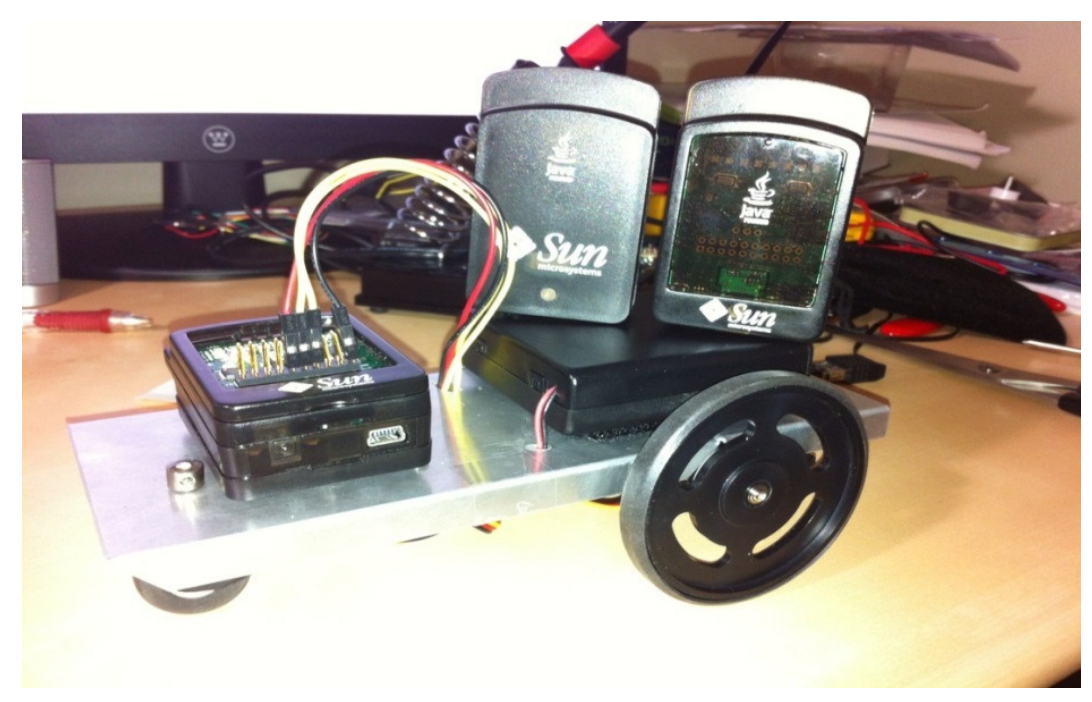

Figure 5: Free Range Sun SPOT on a CAR ${ }^{10}$ 
The Free Range Sun SPOTs connect to each other through a radiogram protocol from the RadiogramConnection interface. The protocol is a datagram-based protocol that allows the exchange of packets between the two devices. Following is a snippet of how to use this communication $^{10}$ :

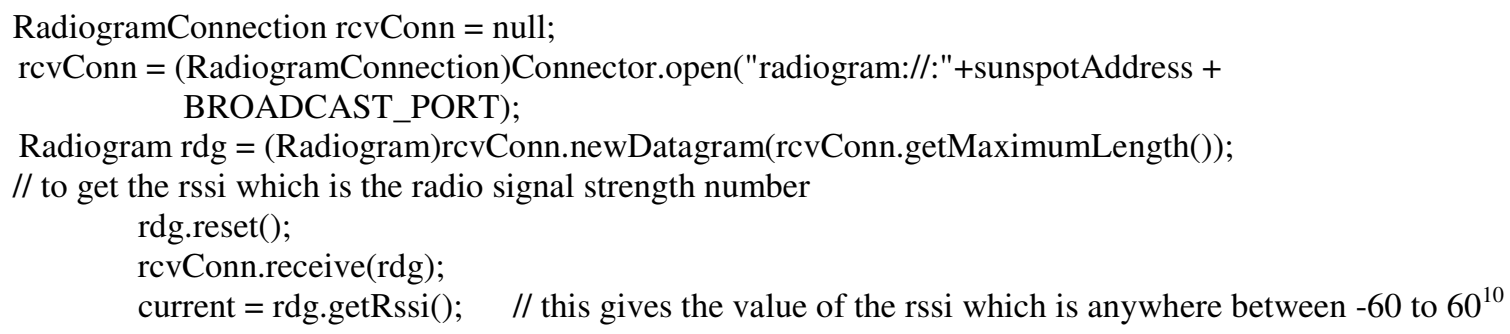

On the hidden Sun SPOT, an application called Controller.java is developed. This application continuously send packets through a radiogram connection to the tracking Sun SPOT. The Sun SPOT controlling the car movement has an application called ServoCar.java which checks to see if any packets are received. This application has a function that returns an integer which is the RSSI (Received Signal Strength Indicator) number that will help determine in which the direction the car should move. The larger the RSSI number, the closer the Sun SPOTs are to each other. It will keep track of the last value and measure it again. If the new value is greater than the previous one, it will move forward. Otherwise, it will turn back. The car will have found its target when the RSSI value of 26 or greater has been found. In this application, there are also two functions that are taking temperature values and light sensitivity and reporting it back to the server through the basestation. The temperature is printed on the server every 5 seconds and light intensity every 7 seconds.

This project was successful and the student commented after finishing her project that "I feel that I have succeeded in what I originally set out to do, which was to simulate a WSN. Although the objective of my simulation (the tracking of one sunspot to another) is not as accurate as I had hoped it to be due to the RSSI signal not being as accurate, I was still able to communicate information from one sunspot to another (in the form of packets being sent back and forth through the radiogram) and I was able to relay sensor readings to a server through the basestation. I also gained some experience in building and designing a robot (given it was just a simple car) but now I feel more comfortable that I can undertake bigger projects." 10

\section{Second Project: Implementation of Wireless Sensor Networks}

The purpose of this project was to design and implement a Wireless Sensor Network. In this design, five motes were used. One mote functioned as the coordinator, three as Routers and one as End Device as shown in Figure 6. 


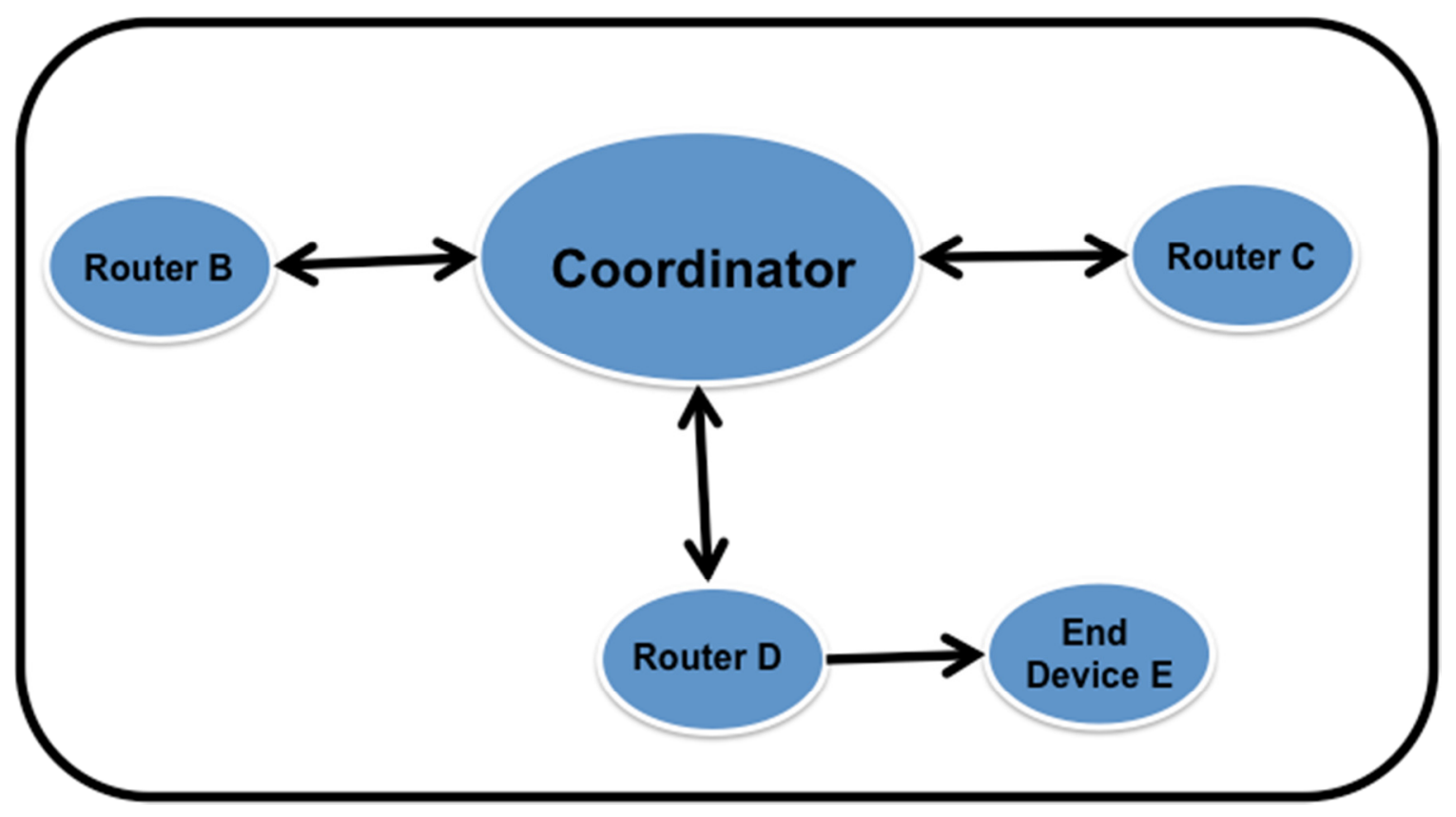

Figure 6: System Diagram ${ }^{11}$

The coordinator consisted of one Arduino Uno, one XBee module (a radio module Zigbee), two TMP36 temperature sensors and three push button switches which are used to send debug data from the coordinator to the other XBee motes as shown in Figure $7^{11}$.

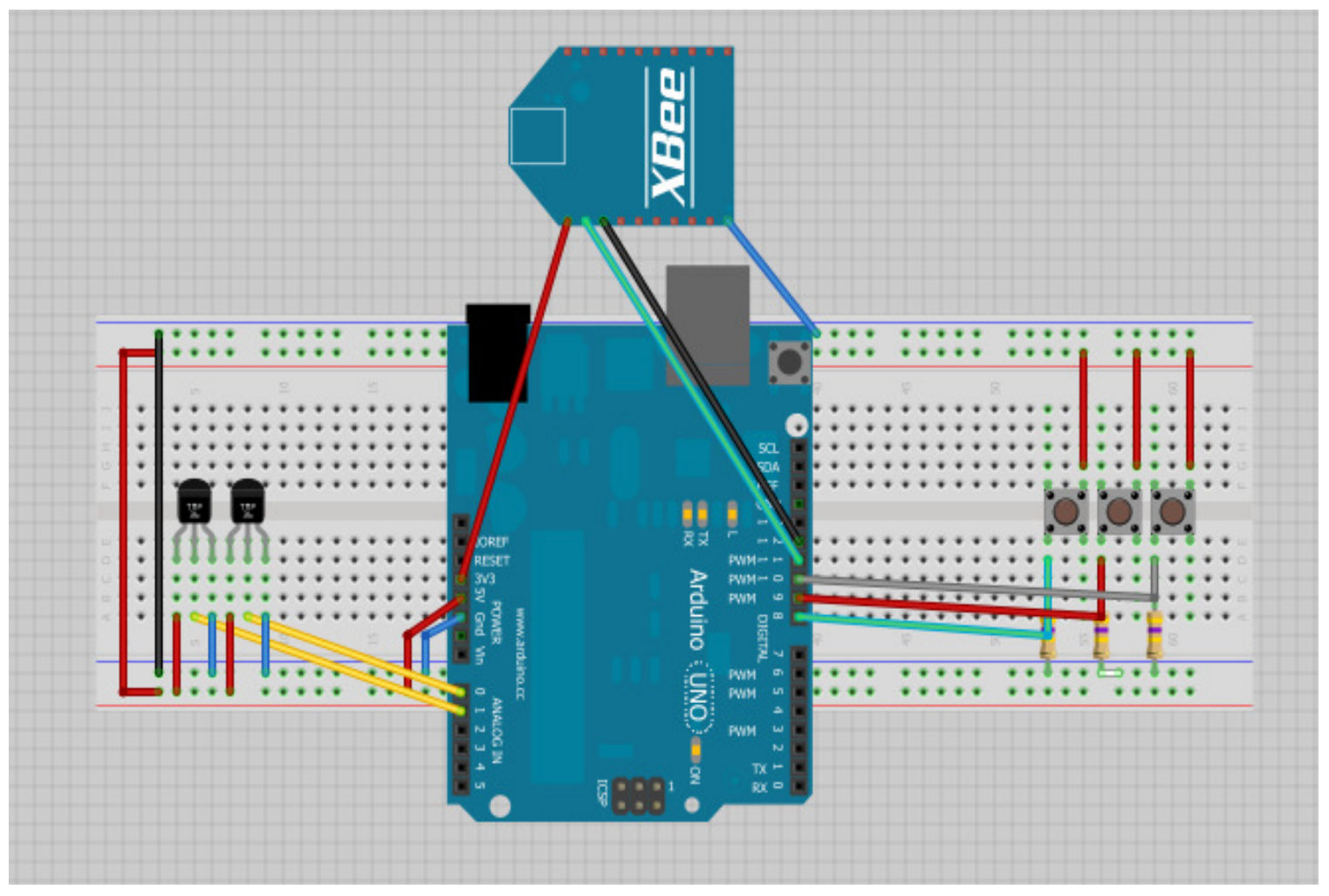

Figure 7: The Coordinator ${ }^{11}$ 
When the push buttons are pressed, one of the three always-on routers receives a packet which tells the XBee device to assert one of the digital I/O pins. Connected to that pin is a LED (See Below). This allows me to check if the network is configured properly and all of the motes can communicate with the coordinator. The coordinator is the only device that is connected to a battery.

The Arduino Uno is in charge of processing all of the data from the network. Since the network is configured in API mode, packets are used to send information. The Arduino then parses the packets and determines what actions are needed to be taken. If it receives digital I/O data from one of the PIR sensors it checks the packet field where digital I/O data is set. If it contains data then we know that one of the PIR sensors was tripped and the Arduino will write out "Mote X was tripped" to the serial bus where $\mathrm{X}$ is the mote that sent the packet. (The mote serial number is extracted from the packet and compared to a list of active motes with their corresponding label). Schematic for The coordinator is given in Figure 8.

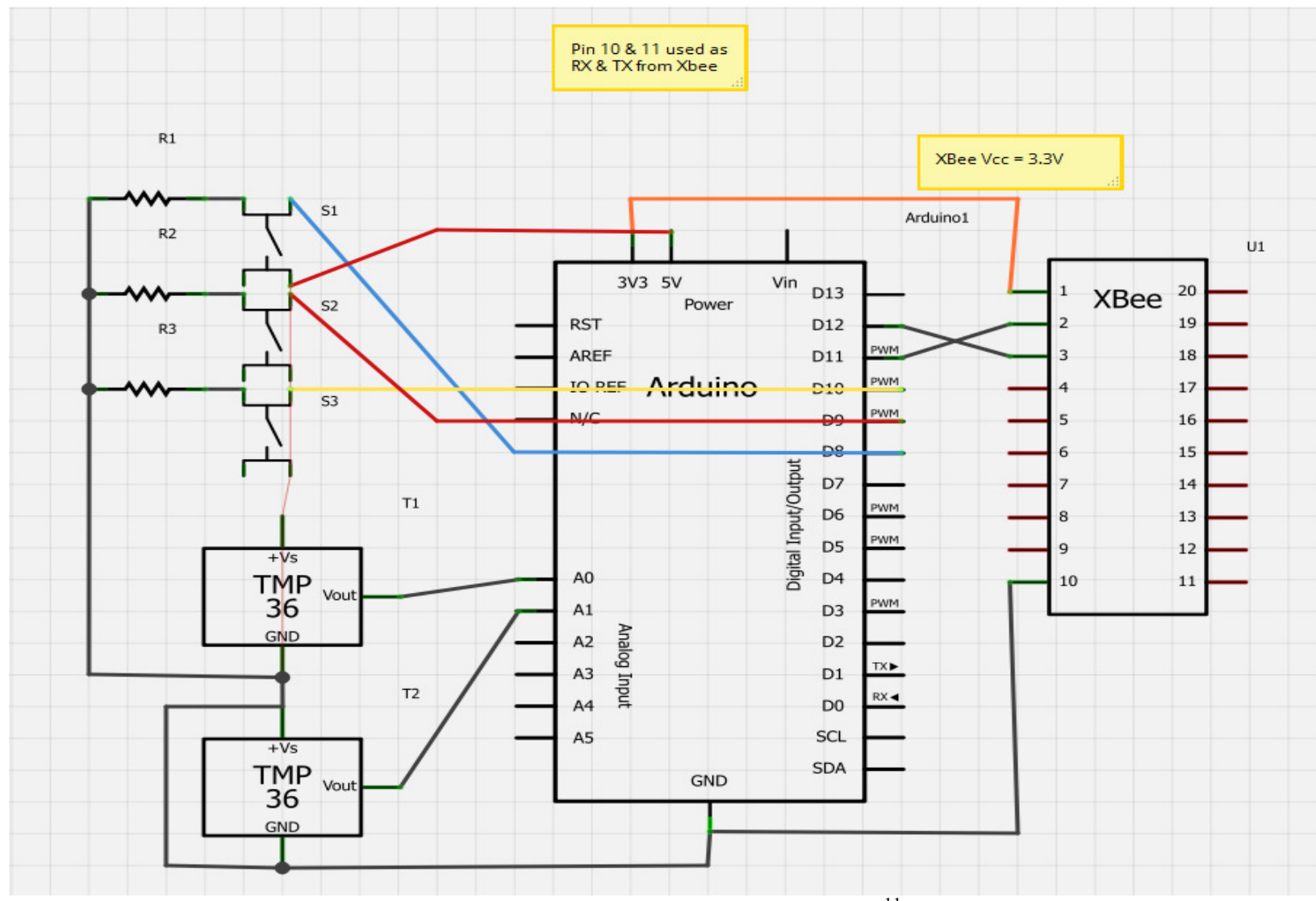

Figure 8: Schematic for the Coordinator ${ }^{11}$

Three motes functioned as Routers in this network. These Routers were in charge of routing all the data that comes from an external device to the coordinator as well as monitoring a PIR (Passive Infrared) sensor that is connected to it (Figure 9). The Routers also listen for incoming packets that is addressed to them. 


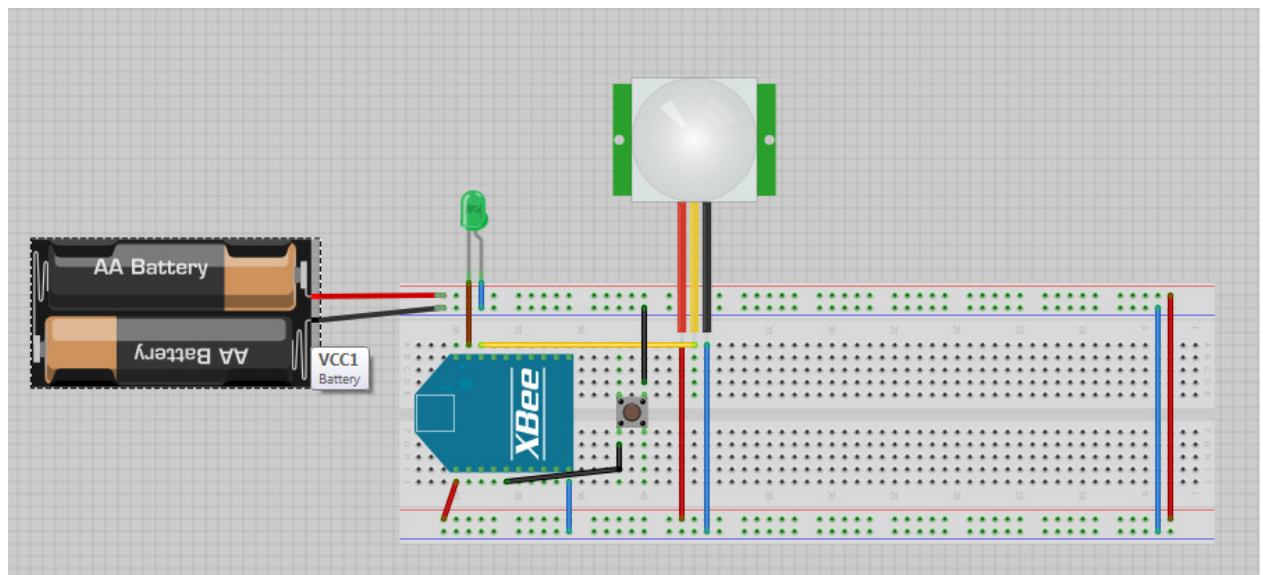

Figure 9: The Router ${ }^{11}$

Along with the PIR sensor, there is also a status LED connected to the XBee. This LED is triggered by the Coordinator when a specific I/O packet is received from the Coordinator. This packet tells the device to turn on the LED that is connected to the Router. This allows the user to visually see when the network has been configured correctly with a simple push of a button (See Coordinator). Figure 10 shows the schematic for one of the Routers.

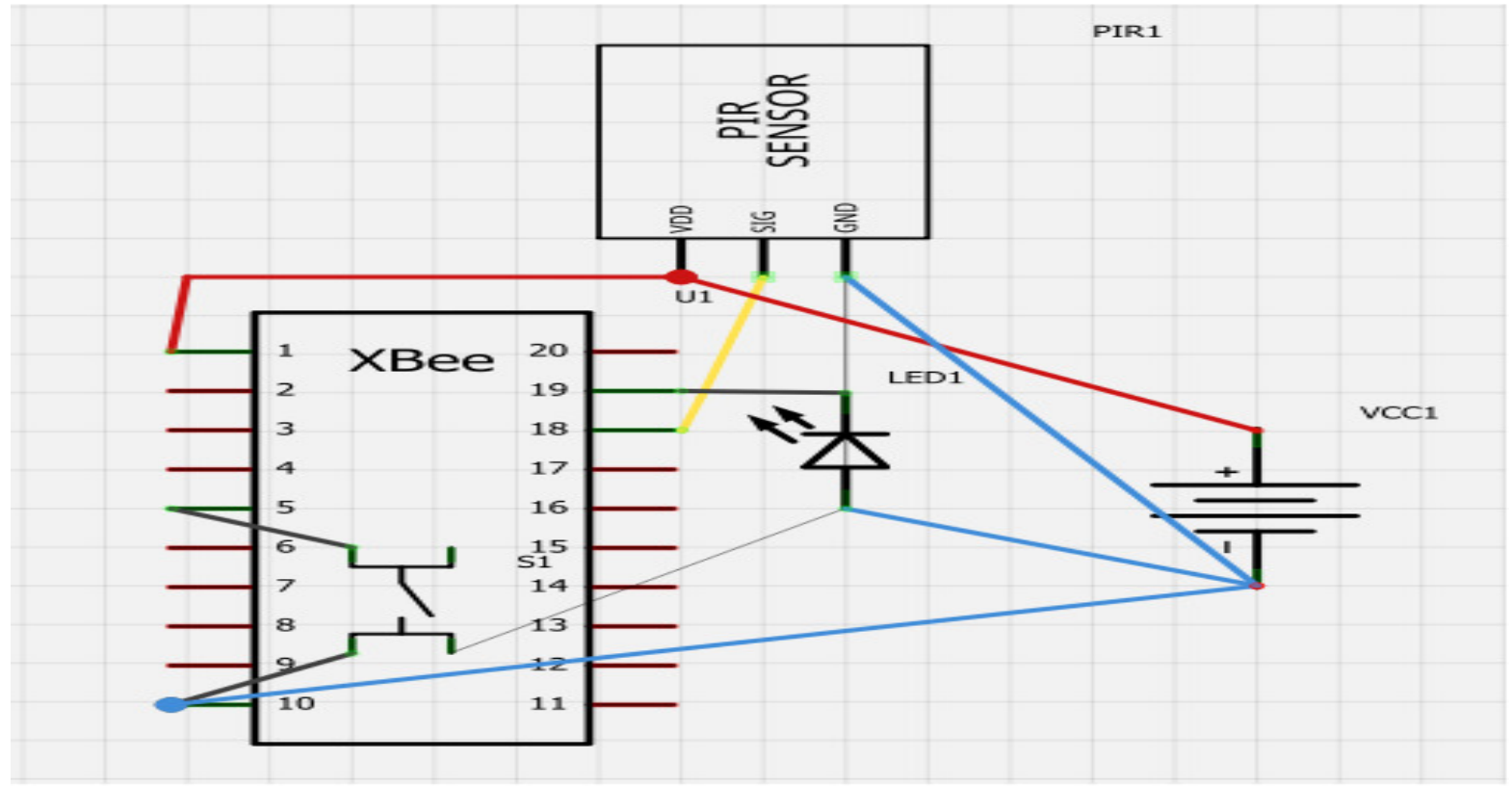

Figure 10: The Router's Schematic ${ }^{11}$

In this network only one End Device was implemented Figure 11). The End Device is in charge of sending temperature data to a router which is then propagated through the network until it eventually reaches the Coordinator. The End Device is extremely power efficient and will run on AA batteries for months to years without requiring change. 


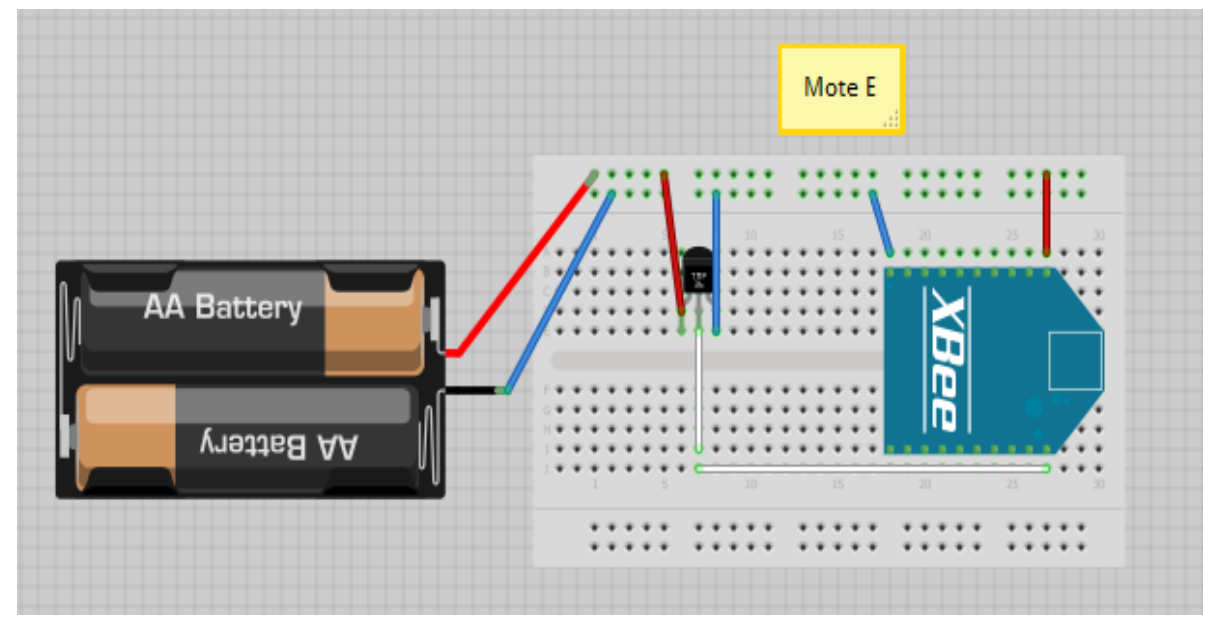

Figure 11: The End Device ${ }^{11}$

The End Device is configured in a way that allows the XBee device to sleep for a minute and ten seconds, wake up for ten milliseconds to check the tmp36 sensor and send out data and then go back to sleep. This allows the device to be on a total of 16 seconds in a period of 24 hours. The schematic for this device is given in Figure $12^{11}$.

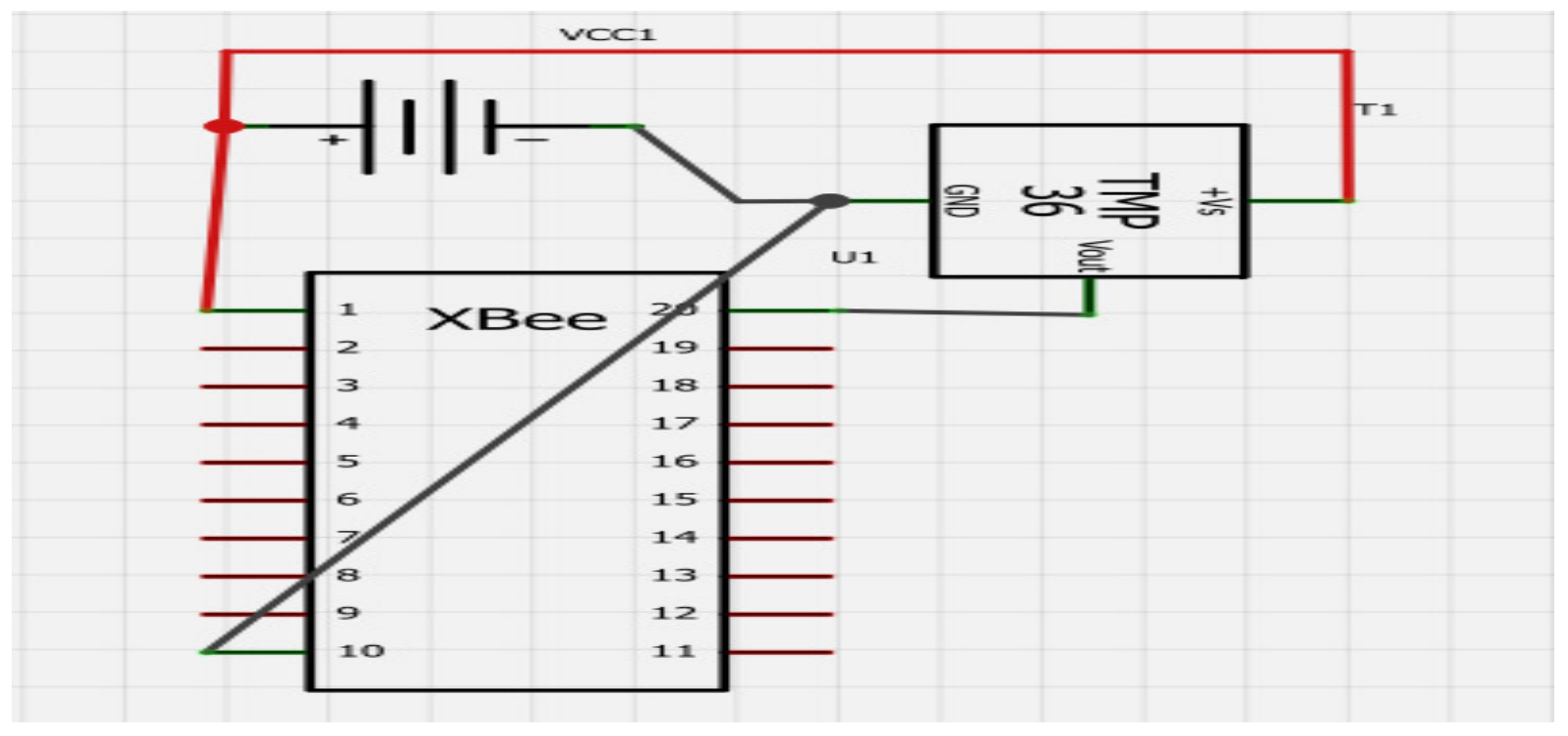

Figure 12: Schematic for the End Device ${ }^{11}$

This project was successful and the student's comment after finishing his project was positive.

\section{Summary and Concluding Remarks}

Wireless sensor networks are a key technology for ubiquitous systems. The future widespread availability of wireless sensor networks entails application designers and embedded engineers to be familiar with it and its emerging standards. The integration of wireless sensor networks concepts in undergraduate computer engineering programs have started in many universities worldwide $^{8}$. To integrate wireless sensor networks concepts into our computer engineering curriculum, we have decided to integrate wireless sensor concepts into our Embedded Systems 
Design II course. In this course, our students are required to do a research paper in an area related to embedded systems design. Recently, many of our students have decided to do their research paper on the topic of Wireless Sensor Networks. As a result of this assignment, many of our students have started to do their senior design project on this subject as well.

This paper discussed recent senior design projects in the area of Wireless Sensor Networks that have been done in our undergraduate senior design course. Our senior design course is structured as a collection of independent student projects. Students find this course both challenging and rewarding as they are required to design, build and troubleshoot a fully functional embedded project. These projects give the students the chance to use their technical expertise and knowledge gained during their years of study. Students work very hard to have a working project by the end of the semester. These projects provide students many opportunities to engage in selfdirected learning. They develop the ability to debug, seek and find information they need, and the ability to understand and reverse-engineer poorly written documentation. The students' feedback and their final project presentation indicate that they have pride in their project accomplishments and have gained confidence in their engineering abilities.

\section{References}

1. Farrugia, Liam, “Wireless Sensor Networks”, Nova Science Publishers, Inc., 2011.

2. Akyildiz, Ian and Mehmet Can Vuran, "Wireless Sensor Networks", Wiley, 2010.

3. Li, Yingshu, My Thai, and Weili Wu, "Wireless Sensor Networks and Applications", Springer, 2008.

4. Dargie, Waltenegus, and Christian Poellabauer, "Fundamentals of Wireless Sensor Networks: Theory and Practice", Wiley, 2010.

5. She, James, and John Yeow, "Nano-Technology-Enabled Wireless Sensor Networks: Overcoming the Limitations from a Device Perspective", Proceedings of the IEEE International on Mechatronics \& Automation, Niagara Falls, Canada, July 2005.

6. Jin, Hai, et al., "Handbook of Research on Developments and Trends in Wireless Sensor Networks: from Principle to Practice”, Information Science Reference, 2010.

7. Forster, Anna and Mehdi Jazayeri, "Hands-on Approach to Teaching Wireless Sensor Networks at the Undergraduate Level”, ITiCSE 10, June 26-30, Bilkent, Ankara, Turkey, 2010.

8. Minaie, Afsaneh, et al., "Integration of Wireless Sensor Networks in the Computer Science and Engineering Curricula", Proceedings of the ASEE Annual Conference, June 2012.

9. Sun SPOT World, Sun SPOT Tutorials, http://sunspotworld.com/Tutorial/index.html, Accessed on 1/2/2014.

10. Zambrano, Gabriela, EENG 4800, Utah Valley University, Final Report, April 2012. 
11. Atkinson, Kimo, "Implementation of Wireless Sensor Networks", EENG 4800, Utah Valley University, Final Report, April 2013.

12. Minaie, Afsaneh and Reza Sanati-Mehrizy, "Embedded Systems Capstone Projects in the Computer Engineering Area of Specialization within the Computer Science Department", Proceedings of 2009 American Society for Engineering Education (ASEE). 